$\begin{array}{llllllllll}E & D & I & T & O & R & I & A & L\end{array}$

\title{
Palobra 18
}

1 ALOBRA, PALABRA QUE OBRA, en su edición 18, fiel a sus principios de calidad editorial y reivindicando su compromiso de atender los requerimientos editorialistas de los sistemas de información, indexación de revistas colombianas de ciencia, tecnología e innovación, se mantiene abierta a las exigencias de valoración de su material académico con miras a su inclusión en las bases de datos internacionales y seguir apostándole al reconocimiento de su calidad científica.

Para la Universidad de Cartagena, en sus procesos de Acreditación de Alta Calidad, PALOBRA, la revista de la Facultad de Ciencias Sociales y Educación se constituyó en un referente destacado en el medio regional y nacional, siendo una de las dos revistas científicas que alcanzaron el reconocimiento en la Base Bibliográfica Nacional-IBBN y el índice Bibliográfico Publindex-IBN, en la categoría B.

Para esta decimoctava edición, se recepcionaron 64 artículos, dentro de los cuales luego de su evaluación externa bajo la modalidad doble ciego por pares académicos reconocidos, se seleccionaron con rigurosidad 19, correspondientes a 8 de investigación científica y tecnológica y 11 de reflexión, relacionados y clasificados según categorías de análisis y referencias, en cuatro áreas temáticas vigentes y oportunas en el actual contexto nacional e internacional: 1. Trabajo social, familia y migraciones, 2. Educación e investigación, 3. Arte, música, carnaval y mediación cultural, y 4 . Corrientes psicoanalíticas y foucaultianas para comprender el amor y la política.

El primer eje temático, Trabajo social, familia y migraciones, lo constituyen 6 artículos de investigación y reflexión, enfocados en temáticas persistentes en la historia de la Revista desde su fundación, cercanas a las líneas e intereses investigativos y docentes de la comunidad académica de la Facultad de Ciencias Sociales y Educación, donde fue fundada y ha sido sostenida desde entonces a hoy. Se desarrollan asuntos sobre las dinámicas de las discapacidades, los profesionales de Trabajo Social en los espacios burocráticos, los contextos familiares rurales, y a su vez a temas vinculados con los lazos transnacionales de las familias.

La psicóloga, terapeuta familiar sistémica y docente investigadora, Martha Silva Pertuz, nos envía el articulo de investigación científica denominado "TRES 
MIRADAS SOBRE LA VEJEZ: DESDE EL PSICOANÁLISIS, LA GERONTAGOGÍA y LA EDUCACIÓN SOCIAL". Trata de analizar los aportes que, desde la tríada de perspectivas del psicoanálisis, la gerontagogía y la educación social, se concretan significativas posibilidades para comprender la vejez en tanto etapa cimera del ciclo vital humano. Se elabora desde una perspectiva interpretativa que pretende adentrarse en el significado de las acciones humanas y de la práctica social relacionadas con la vejez. Se estructura el artículo en la intencionalidad de la comprensión, la significación y la acción activado por un persistente interrogante: ¿Qué perspectivas y posibilidades se ofrecen para la comprensión de la vejez desde los aportes del psicoanálisis, la gerontagogía y la educación social? Los hallazgos, siempre parciales de la autora, permiten aproximaciones positivas -que no positivistas-, humanas y respetuosas de la complejidad humana, en particular, de la vejez.

Un segundo artículo, resultado de reflexión, es enviado por la trabajadora social y candidata a doctora en Ciencias Sociales de la Universidad Nacional General Sarmiento de Buenos Aires, Argentina, Claudia Bermúdez Peña, titulado "PROFESIONALES DE TRABAJO SOCIAL EN LA BUROCRACIA DE $L A S$ NACIONES UNIDAS". La autora se propone visibilizar y describir la participación de organizaciones de Trabajo Social y de trabajadores sociales como parte de la burocracia del sistema de las Naciones Unidas. En este sentido, muestra, por un lado, la configuración de la disciplina/profesión en el marco de unas relaciones globales que la hicieron posible $y$, por otro, la manera como llegó a América Latina la profesión. El periodo de tiempo que enmarca el análisis comprende dos décadas que van desde el surgimiento de las Naciones Unidas en el año 1945, teniendo en cuenta que desde ese momento organizaciones de profesionales de Trabajo Social se vincularon de manera activa como organismos no gubernamentales, hasta la década de los sesenta, momento de ruptura que colectivos de trabajadores sociales en Latinoamérica plantearon con el perfil de profesionales de Trabajo Social creados desde la ONU. Un vector transversal para el análisis, lo constituye el reconocer a este sistema intergubernamental como un escenario proveedor de empleo para trabajadores sociales y la influencia de esa condición en los desarrollos de la disciplina/profesión.

El tercero, se denomina "LAS FAMILIAS RURALES, SUJETOS COLECTIVOS DE DERECHOS. EL CASO DE GUAYABAL DE SÍQUIMA", es resultado producto de investigación de las autoras Ana Marcela Bueno, Trabajadora Social y doctoranda en Trabajo Social de la Universidad Nacional de Rosario, Argentina 
y Miryam Fernanda Torres G. Trabajadora Social y doctoranda en Ciencias de la Educación Universidad Pedagógica y Tecnológica de Colombia, ambas docentes investigadoras de la Universidad de La Salle, Bogotá. Las autoras documentan las dinámicas sociofamiliares de Guayabal de Síquima, en Colombia, a partir de su abordaje como sujeto colectivo de derechos, reconociendo sus relaciones cotidianas, condiciones socioeconómicas y culturales, además de dar cuenta de la vivencia de la participación como una concienciación respecto a la reivindicación de sus derechos en un ámbito tradicional como el contexto rural. Lo anterior se realizó a partir de un estudio cualitativo recolectando información a través de una encuesta inicial y su complementación con entrevistas y talleres, con la participación de padres, madres, niños y niñas, quienes desde sus experiencias expresaron vivencias referidas a sus derechos como colectivos familiares. Como conclusión general se establece que identificar esta condición de las familias, favorece la sociedad que se viene construyendo hacia la paz, la cual requiere de sujetos empoderados de sus derechos y por ende conscientes de sus posibilidades como sujetos y colectivos en el ejercicio de su ciudadanía.

El cuarto articulo, perfilado desde las migraciones, es un producto resultado de reflexión de la autoría de Orlando Pertuz de Ávila, docente investigador del Instituto Internacional de Estudios del Caribe de la Universidad de Cartagena, titulado "LAZOS TRANSNACIONALES: LA MIGRACIÓN COLOMBIANA A VENEZUELA Y EL DESARROLLO DE LA VIVIENDA POPULAR EN CARTAGENA, 1973-1983". Durante los años de la bonanza petrolera, miles de colombianos, incluyendo un buen número de cartageneros, emigraron a Venezuela en procura de mejores oportunidades. Una vez establecidos enviaron remesas que fueron invertidas por sus familias en la mejora de sus viviendas. A largo plazo, el envío de remesas y la capacidad de auto-gestión de los sectores populares ayudaron al desarrollo del parque de viviendas de la ciudad. Este artículo plantea que las remesas enviadas desde Venezuela fueron un factor transnacional que contribuyó a la evolución urbanística de Cartagena. Así mismo, los inmigrantes cartageneros obraron como sujetos transnacionales capaces de incidir en el desarrollo histórico la ciudad a pesar de la distancia, y su legado se convirtió en materia de debate entre académicos vinculados a organismos transnacionales.

El quinto articulo recibido, se denomina "DIFUSIÓN DE DRAMAS COREANOS, UN ANÁLISIS DE SU ÉXODO A AMÉRICA LATINA Y COLOMBIA”, de la autora Liz Andrea Zarco Quintero, comunicadora social de la Universidad de Cartagena 
y miembro del semillero de investigación "Comunicación, Educación y Cultura" adscrito a la Facultad de Ciencias Sociales y Educación de la misma institución. El articulo realiza un análisis de la difusión de los dramas coreanos en América Latina y Colombia, partiendo de una breve descripción del movimiento Hallyu, de origen asiático. Aunque se establece que las relaciones diplomáticas, las relaciones comerciales y los procesos migratorios fueron factores determinantes que hacen posible su llegada al continente, se plantea la hipótesis de que estos no aplican para el caso de Colombia.

Y el sexto articulo incluido en este eje temático, nos lo envían las estudiantes de Trabajo Social de la Universidad de Cartagena, Angie Paola Menco Cadena y Diana Paola Cantor Rueda, artículo producto de reflexión, titulado, "DINÁMICAS DE LA DISCAPACIDAD PENSADAS DESDE EL LUGAR DE LAS FAMILIAS Y/O CUIDADORAS/ES: EXPERIENCIAS COMO EJE DE REFLEXIÓN PARA LA INCLUSIÓN". Las autoras traen a discusión las dinámicas familiares de las personas con discapacidad, con énfasis en las que se refieren a los roles y prácticas de las cuidadoras y los cuidadores, instalando como referente la normativa que respalda y garantiza los derechos de dicha población. De acuerdo al planteamiento teórico, se realiza una aproximación a las realidades cotidianas vividas por las personas con discapacidad, abordando casos específicos en lo comunitario y en lo organizacional. Además, se analizan las apuestas que se llevan a cabo en el Trabajo Social frente a la articulación de organismos, instituciones y sociedad para propiciar el fortalecimiento de las relaciones interpersonales de las personas con discapacidad, atendiendo a la socialización directa con su familia y su entorno. Por último, se evidencia que las experiencias en el marco de otra mirada de la discapacidad albergan la labor de las/os cuidadoras/es en el desarrollo personal y social de las personas con discapacidad; no obstante, este cuidado tiende a sobreponer la vida de la persona con discapacidad sobre las expectativas personales, sueños, deseos, necesidades, temores y aspiraciones de las/os cuidadoras/es.

En el segundo eje temático Educación e investigación, se incluyeron 7 artículos de investigación y reflexión, en los cuales se desarrollan temáticas sobre historia de la educación, gestos de la descolonización y que pasado narrar, pertinencia de la concepción de lectura, prácticas pedagógicas, cátedra local, historia de la educación y el pensamiento de los profesores.

En primera instancia los resultados de investigación del artículo "LOS PENSAMIENTOS DE LOS PROFESORES UNIVERSITARIOS DE CIENCIAS DE LA 
SALUD. CONCEPCIONES SOBRE ENSEÑANZA Y APRENDIZAJE", realizado por la magíster en Educación de la Universidad de Córdoba y estudiante de Doctorado en Ciencias de la Educación de la Universidad de Cartagena Carolina Castellanos Ramos. El artículo está basado en los resultados de la investigación referida al pensamiento docente y su quehacer pedagógico de los profesores universitarios de Ciencias de la Salud, que fueron identificados según el criterio analítico de la triada dimensional propuesta por Dewey (1938), constituidas por lo intelectual, lo personal y lo socioafectivo. Este texto indaga historias de vida, tipos de conocimientos y estatus-roles de los maestros y reconstruye de manera holística la imagen con la que se identifican y son identificados los docentes. Los resultados no solo tributan hacia el conocimiento y reconocimiento de los docentes universitarios, sino que proponen la transformación educativa a partir de la construcción de la identidad docente y la actualización pedagógica.

Por su parte, las docentes Ana María Chinchilla Cogollo del Colegio de las R. R. Esclavas del Sagrado Corazón de Jesús, Yeimmy Carranza Abella del Colegio Cafam Floresta, Dania Acosta López del Colegio Agustiniano Norte y Heidi Mahecha Hernández del Colegio Juan Ramón Jiménez, proponen el artículo de investigación titulado, "PERTINENCIA DE LA CONCEPCIÓN DE LECTURA Y DE SUJETO LECTOR CONTENIDA EN EL PLAN NACIONAL DE LECTURA Y ESCRITURA “LEER MI CUENTO"”. El análisis del Plan Nacional de Lectura y Escritura "Leer es mi cuento", les permite explicitar la concepción de lectura y de sujeto que le subyacen, estableciendo la pertinencia de dichas concepciones para la educación con el propósito de abrir espacios de reflexión y diálogo en el que los maestros pueden tener mayores elementos para problematizar y cuestionar sus prácticas educativas, que se ven permeadas por los discursos gubernamentales que las afectan. De esta manera promueven la resignificación de su ejercicio como sujetos que aportan a la formación lectora y a través de ella a la formación de sujetos críticos que inciden en la transformación de la sociedad.

El tercer articulo resultado de investigación se denomina "PRÁCTICAS PEDAGÓGICAS DE LAS MAESTRAS DE EDUCACIÓN PREESCOLAR CON POBLACIÓN DIVERSA" realizado por las magísteres en Educación con profundización en poblaciones vulnerables del Tecnológico de AntioquiaInstitución Universitaria (TdeA), Luisa Fernanda Caballero Marín, Katherine Ocampo Roldán y Nathaly Restrepo Restrepo. Tienen como objetivo analizar las prácticas pedagógicas que realizan las maestras de educación preescolar con 
población diversa, para lo cual identificaron los discursos y caracterizaron las estrategias de enseñanza a partir de un estudio de caso con tres participantes, según el paradigma cualitativo y el enfoque interpretativo. Como resultados se encontró que las prácticas pedagógicas de cada maestra son diferenciadas, que parten de su experiencia, su formación y la relación que configuran frente al otro. Dentro de las estrategias más utilizadas para atender la diversidad se resaltan las estrategias centradas en el medio, en la autorregulación, actividades rectoras, demostrando coherencia entre algunas afirmaciones y acciones pedagógicas. Se denota, sin embargo, falta de implementación de estrategias centradas en el estudiante, en el desarrollo de la autonomía, en el reconocimiento de las capacidades, en las habilidades para la solución de problemas y que promuevan los procesos socioemocionales.

El cuarto, se denomina "LA CÁTEDRA LOCAL COMO ESTRATEGIA DE AULA PARA FORMAR IDENTIDAD HISTÓRICA Y CULTURAL", producto de investigación de la autoría de Yolima Rodríguez Ramírez, docente de la Institución Educativa Dorada, La Dorada, Caldas; licenciada en ciencias sociales y aspirante a magíster de historia de la Universidad Tecnológica de Pereira. El artículo busca resaltar la importancia de la historia barrial y local del municipio de La Dorada desde un ejercicio investigativo formativo en la Institución Educativa Dorada, donde los estudiantes del grado séptimo y once recolectaron diversas fuentes de información y desarrollaron diferentes actividades de exposición de sus resultados para así dar a comprender a su comunidad educativa su propia cotidianidad y su visión de la historia local. La escuela, se muestra en este caso concreto como artífice de la reconstrucción de la historia local desde sus fuentes activas, en donde los estudiantes comprometidos con la investigación histórica hacen un juicioso ejercicio de reconstrucción de la memoria colectiva oral, la de sus vecinos, familiares y conciudadanos que les permitieron redescubrir su pasado $\mathrm{y}$, de paso, propiciar nuevas dinámicas a la coyuntura social de la localidad.

Formando parte de este eje, el quinto artículo pertenece a las autoras Elizabeth Torres Puentes, magíster en educación de la Universidad Pedagógica Nacional y estudiante del Doctorado interinstitucional en Educación, Yeimmy Cárdenas Palermo, magíster en educación de la Universidad Pedagógica Nacional y Doctora en Educación del Programa Interinstitucional de las universidades del Valle, Distrital Francisco José de Caldas y Pedagógica Nacional y Carol Pertuz Bedoya, licenciada en Psicología y Pedagogía y magíster en Estudios en Infancias de la Universidad Pedagógica Nacional (Colombia), las que 
enviaron el articulo de investigación denominado, "LA INFANCIA Y LOS NIÑOS EN TIEMPOS DE GUERRA: EL CASO DE NICARAGUA, EL SALVADOR $Y$ GUATEMALA", en el cual presentan los resultados de la investigación "Infancia, educación y posacuerdos: una mirada a los casos de Nicaragua, El Salvador y Guatemala", financiada por el Centro de Investigaciones de la Universidad Pedagógica Nacional (CIUP), durante la vigencia 2017. Se recorre la pregunta por los discursos que circularon sobre la infancia y los niños durante las décadas de los años ochenta en el marco de los conflictos armados centroamericanos, los cuales comparten una historia de guerras civiles y movimientos revolucionarios, atravesados por las dinámicas de la Guerra Fría.

El sexto articulo, es presentado por la docente investigadora Muriel Vanegas Beltrán, profesora asociada de la Universidad de Cartagena - Instituto Internacional de Estudios del Caribe, doctora en Ciencias de la Educación y magíster en Estudios del Caribe; quien asume como objeto de reflexión " $L A$ EDUCACIÓN PÚBLICA DEL DEPARTAMENTO BOLÍVAR DURANTE LA PRIMERA MITAD DEL SIGLO XX". Estudia la Educación Pública del departamento de Bolívar durante la primera mitad del siglo XX. Se ocupa de analizar la recepción e implementación de las reformas educativas en la primera mitad del siglo $\mathrm{XX}$ en las regiones de provincia y su articulación con los municipios de la costa Caribe colombiana. Con base en fuentes primarias, en el marco de la legislación educativa contemporánea así como en informes de directores de instrucción pública y de ministros de educación, se construye una rigurosa aproximación a las coyunturas en las que se ponen de relieve las tensiones del difícil proceso de modernización del sistema educativo a nivel nacional, en contraste con las precarias condiciones sociales y económicas que imperaban en ese período en el departamento, focalizando la mirada en las actuaciones de las autoridades educativas locales de acuerdo a las disposiciones normativas que emanaban desde el nivel nacional.

Y el séptimo articulo de este eje, es el resultado de reflexión, "¿QUÉ PASADO NARRAR EN LA EDUCACIÓN? GESTOS DESCOLONIALES EN LA HISTORIA DEL BACHILLERATO ARGENTINO", de Francisco Ramallo, investigador y docente de la Facultad de Humanidades de la Universidad Nacional de Mar del Plata. Se constituye en el rastreo de una conversación entre las formas de componer la historia de la educación y las trasgresiones que podrían enunciarse desde las perspectivas descoloniales. En un primer momento destaca una narrativa genealógica de estas perspectivas en la educación, convocando a 
la recuperación de nuestro propio paisaje colonial, desde la (des)composición de "el" relato universal. En un segundo momento del desarrollo del texto aquí incluido, los relatos del bachillerato argentino de la segunda mitad del siglo XX son interpelados a partir de los hallazgos de la investigación doctoral del autor en la que propone abandonar una enunciación nostálgica que se expresa en narraciones utópicas de los pasados en la educación. Esta preocupación desemboca en la necesidad de reconocer gestos oportunos para significar otros hilos de sentidos capaces de intervenir el relato colonial de la educación que se habitan cotidianamente.

El tercer eje temático de esta decimoctava edición de PALOBRA, se articula con un conjunto de artículos que abordan temáticas alrededor del $\underline{\text { Arte, música, }}$ carnaval y mediación cultural.

El primer artículo de reflexión del eje, se denomina, "LOS LENGUAJES DE LA MÚSICA UN EJE TRANSVERSAL DEL CURRÍCULUM", del doctor en ciencias de la Educación, de la Universidad de Caldas, Manizales, Manuel Antonio Pérez Herrera. Para el autor el currículum se devela como eslabón entre la investigación formativa y equilibrada de códigos universales y códigos del contexto. Acápite científico curricular que permite ampliar el marco de posibilidades de acceder a nuevos conocimientos científicos-socio- culturales, con identidad, y en procura de mantener la comunicación dialógica constante entre los lenguajes musicales, la investigación y la producción musical en contextos. Propuesta que se concreta a través de la puesta en marcha de una didáctica constructivista alternativa que integre prácticas y saberes en un currículo comprensivo, atendiendo así propósitos como los de encontrar equilibrio en los significados de la música desde una práctica pedagógica mediadora del pensar, sentir y hacer de los sujetos cognoscentes, quienes comprenden por qué la música se constituye en lenguaje que transversaliza lo humano y lo científico.

Por su parte, el segundo artículo de reflexión "LAS ARQUITECTURAS DE LAS PRÁCTICAS EN LAS ARTES VISUALES. CUESTIONES PARA PENSAR OTRAS $P R A ́ C T I C A S^{\prime \prime}$, fundado en trabajo doctoral de la autora, Flor Ángela Castro, mediante la apropiación y desarrollo de presupuestos epistemológicos de la práctica pedagógica en medio de las arquitecturas de las prácticas y la práctica de las artes visuales, provoca otros significados y sentidos a la construcción del discurso relacionado con los nuevos modos de comunicación en la escuela. A partir de estas dos categorías que estructuran el tópico objeto de reflexión del 
presente texto se busca esclarecer relaciones posibles que permitan un primer acercamiento a la utilidad de la teoría en construcción, que se sintetizan en dos proposiciones. La primera, que es necesario comprender los discursos, haceres y relaciones en que ha emergido la práctica de las artes visuales para llegar a la construcción de nuevas y buenas prácticas. Y la segunda, que las buenas prácticas forjadas desde las nuevas condiciones cultural-discursivas, material-económicas y político-sociales están atravesadas por dos categorías imprescindibles en la configuración de sujetos de bien: la experiencia subjetiva y la experiencia estética. El texto concluye con una provocación para replantear desde la relación cultura visual-artes visuales, nuevas prácticas pedagógicas basadas en una estructura de buenas prácticas reconocidas como lo cambiado en lo que se hace.

El tercer articulo, denominado, "MÁS ALLÁ DE LA FIESTA: PARTICIPACIÓN DE NIÑOS Y NIÑAS EN EL CARNAVAL DE RIOSUCIO", producto de investigación, de las investigadoras Gloria Abimeleth Ardila Guerrero ingeniera de alimentos, candidata a magíster en Educación y Desarrollo Humano, Universidad de Manizales, Eliana Cristina Medina Ramírez Socióloga, candidata a magíster en Educación y Desarrollo Humano Universidad de Manizales y Yudy Viviana Medina Ramírez Licenciada en Tecnología Informática, candidata a magíster en Educación y Desarrollo Humano Universidad de Manizales. Presentan y analizan las diferentes formas de participación infantil y las subjetividades políticas de niños y niñas que emergen en el Carnaval de Riosucio (Colombia). La investigación que da origen a este artículo: "La participación infantil en los ritos carnavalescos de los niños y niñas, una experiencia de subjetividad política", se centra en la importancia de hacer visible la participación de niñas y niños en los ritos festivos propios del carnaval, evidenciando la configuración de subjetividad política a partir de dicha participación, abriendo un espectro de posibilidades que permiten otras prácticas de sí, un despliegue de configuraciones de subjetividades que hacen visibles y audibles sus voces, sus modos de habitar el pueblo, su manera de ver la realidad en época de carnaval, trascendiendo la hegemonía a la que normalmente son sometidos.

Y el cuarto artículo de este eje, titulado, "iVENIMOS POR LAS VÍRGENES! CERROMATOSO Y EL DIABLO EN URÉ", es producto de reflexión del artista investigador, curador y docente universitario, Manuel Antonio Zúñiga. Muestra como con ocasión del referéndum de 2008, que implicó la redelimitación del territorio de San José de Uré, antiguo palenque, en el departamento de Córdoba, en el Caribe colombiano, se generaron dinámicas 
locales emergentes debido a que como resultado de aquel, este municipio recibiría millonarios recursos de regalías por la explotación del ferroníquel que adelanta la multinacional BHP Billiton, dueño de la mina de Cerromatoso. En medio de las tensiones derivadas del proceso de reordenamiento territorial, de la reconfiguración política y del temor fundado por el accionar violento de grupos al margen de la ley, tuvo lugar el Laboratorio de Mediación Creación "UREDOS_relacional", promovido por el Ministerio de Cultura. En este contexto, el autor relata cómo la comunidad uresana encontró, a través de la movilidad del cuerpo, una mediación cultural como forma de empoderamiento pacifista de resistencia a las tensiones propias de la situación, en medio de viejas y nuevas expresiones de violencia, de desigualdades sociales y económicas.

El último eje temático, denominado Corrientes psicoanalíticas y foucaultianas para comprender el amor y la política, incluye los artículos que desarrollan reflexiones sobre el amor y sobre el racismo foucaultiano como herramienta analítica.

Como espacio de reflexión, el primer artículo de este eje titulado "REFLEXIONES SOBRE EL AMOR EN PSICOANÁLISIS: UNA LECTURA A LA ENSEÑANZA DE FREUD Y LACAN" de las docentes investigadoras de la Universidad Metropolitana de Barranquilla, Colombia, Judith Elena García Manjarrez y Dennys Martínez Franco. Se trata en el presente artículo de discernir, desde la enseñanza que legaran Freud y Lacan, el asunto por el amor y con ello el encuentro y desencuentro con el otro y lo que esto implica para la subjetividad, lugar desde el que cada uno hace lazo social. Las autoras estructuran el texto alrededor de la pregunta por el amor, en tanto ha sido la piedra de toque que ha convocado desde siempre al ser humano, encajado en el centro del vínculo social. Muestran cómo desde la perspectiva problematizadora de estos autores, el amor ha aparecido en nuestra cultura como aquello que de cierta manera da un sentido particular a la vida y como la promesa con la que algunos sujetos esperan encontrarse en un algún momento vital, pero también lo que al tiempo trae consigo trae consigo la infelicidad y el dolor, en tanto se sitúa del lado de la imposibilidad subjetiva y de la queja humana.

Y el segundo articulo de reflexión "EL RACISMO FOUCAULTIANO COMO HERRAMIENTA ANALÍTICA PARA EL DEBATE POLÍTICO CONTEMPORÁNEO", de Carlos Mario Herrera Casadiego, investigador en las líneas temáticas de filosofía política, ciencia política, violencia y conflicto armado, plantea el autor, a partir de la revisión de la obra de Michel Foucault, pone en evidencia cómo el 
concepto de racismo formulado por el filósofo francés puede emplearse como herramienta analítica para el debate político contemporáneo. En el transcurso del cumplimiento de ese propósito pone el autor de relieve los rasgos principales de los tres paradigmas de poder expuestos por Foucault, cuales son el soberano, el disciplinario y el biopolítico. Esta exposición le permite al autor estructurar un marco de referencia para introducir el concepto de racismo, definir sus características y operatividad, así como mostrar casos en los que la categoría puede ser instrumento de análisis para fenómenos políticos concretos.

Para la portada e ilustración artística de la decimoctava edición de Palobra, reconocemos y rendimos homenaje póstumo a la docente Rosa Jiménez Ahumada, trabajadora social, especialista en Administración de Empresas y magíster en educación, por sus 35 años de trabajo entre la docencia, la dirección del Observatorio del Desplazamiento Forzado, la coordinación de la Maestría en Conflicto Social y Construcción de Paz, la jefatura del departamento de posgrados de la Facultad de Ciencias Sociales y Educación, de la Universidad de Cartagena y colaboradora en varias ediciones de la Revista Palobra. Paz en su tumba.

Por último, consideramos importante volver a destacar, como en ocasiones anteriores lo hemos venido expresando, el propósito persistente de los editores y responsables de esta Revista, no solo de sostener nuestra calidad editorial, de forma y de contenido, sino escalarla en cada número hacia la excelencia. Hemos venido cumpliendo y cada vez lo haremos con mayor efectividad, con los parámetros de optimización de los procesos de gestión editorial, de validación de la evaluación y de la visibilidad y el impacto de nuestra revista. A partir del próximo número, de cara a ampliar sustancialmente nuestra visibilidad nacional e internacional, la revista Palobra se transformará también en revista digital, alojada en la página web de la Universidad de Cartagena.

Javier Hernández García

Editor

Dora Piñeres De La Ossa

Editora Invitada 